\title{
COSMIC X-RAY SPECTRA
}

\section{K. A. POUNDS}

Dept. of Physics, University of Leicester, England

Nine years of rocket and balloon observations, considerably enhanced in the last few months by the survey experiment on the Uhuru satellite, have revealed a large number - now approaching 100 - of cosmic x-ray sources, remarkable in both their luminosities $\left(\mathrm{Lx}=10^{36}-10^{46} \mathrm{ergs} \mathrm{s}^{-1}\right)$ and apparent variety. Spectral data, expected eventually to be of crucial importance in revealing the emission processes and dynamics of individual sources, have been of generally inadequate quality to date (e.g. Figure 1).

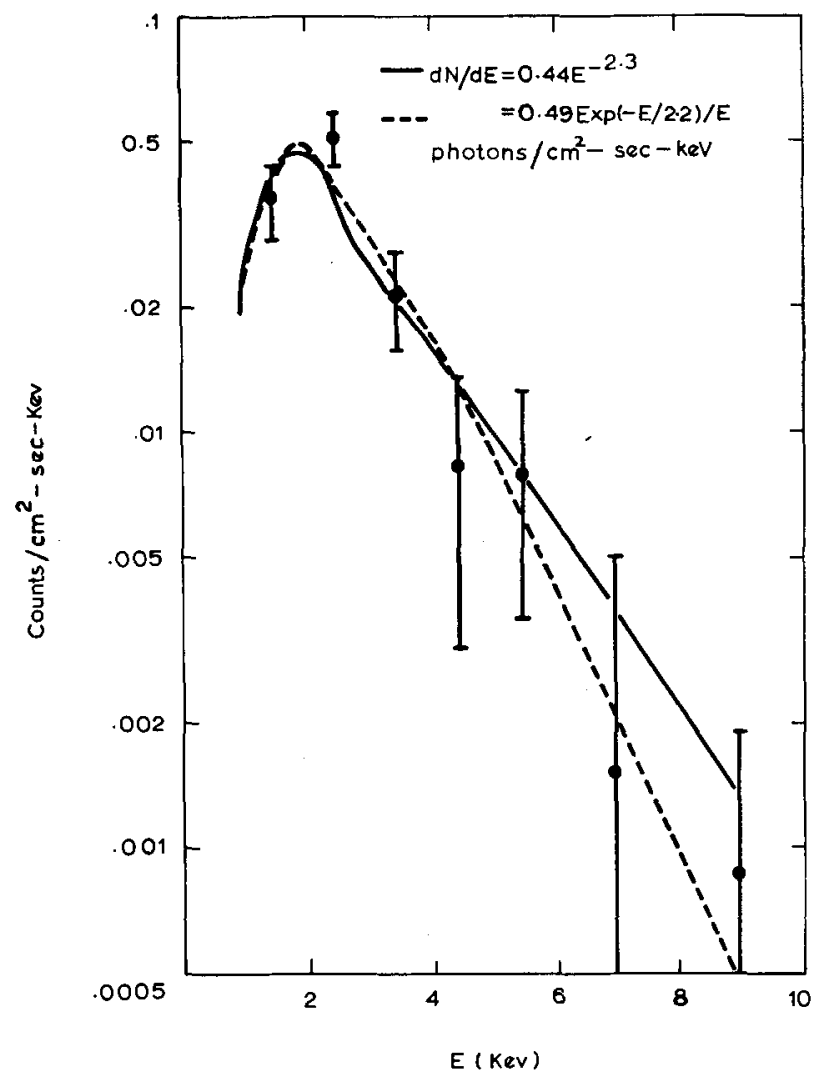

Fig. 1. Counting rate spectrum of SN1572 (Tycho's supernova) obtained on an A.S. \& E proportional counter rocket experiment in December 1968. The continuous and dashed curves are the computed count rate distributions giving the best fit to the observed data points for, respectively, assumed power law and exponential (thermal) spectra. Over the limited bandwidth observed, the available statistics allow an acceptable fit to either spectrum. 
In the main, observations have been made with proportional counters at photon energies below $20 \mathrm{keV}$ and with scintillation counters between 20 and $1000 \mathrm{keV}$. [1] Though both types of detector have a desirably high photon efficiency for observation of the weak cosmic signals, they also have a low intrinsic spectral resolution. Further limitations in the available spectral data on many $x$-ray sources are due to poor statistics and a narrow measured bandwidth. Spectra have generally been compared with either an exponential or power law distribution, with the aim of establishing a thermal bremsstrahlung (exponential) or synchrotron/inverse Compton (power law) emission mechanism. In fact only in a dozen or so cases have spectral observations been of much value as yet in indicating the physical properties of the x-ray source and in no case has the emission mechanism been definitely established. The following account briefly describes the most likely emission processes and then reviews the best of the available data and their interpretation.

\section{Emission Processes}

The detection of $\mathrm{x}$-rays from a cosmic source is usually taken to imply either a large amount of extremely hot gas or a copious flux of relativistic electrons existing in that source. The hot gas source then emits $x$-rays by a combination of thermal processes (bremsstrahlung, recombination radiation and line emission) which may be apparent from an overall exponential shape to the observed x-ray spectrum, or by some nonthermal process. Excluding non-thermal bremsstrahlung, since this is similar in form and would in most cases rapidly lead to thermal emission, the most likely non-thermal processes would involve the interaction of electrons with an ordered magnetic field (synchrotron radiation) or with a local field of low energy photons (inverse Compton radiation). As noted below, these last two mechanisms would generally yield $\mathrm{x}$-ray spectra similar in form to the electron number spectra which, from radio observations, often exhibit a power-law form.

\section{A. THERMAL}

The thermal continuum emission from a source at distance $\mathrm{d} \mathrm{cm}$ and temperature $T \mathrm{~K}$, which is transparent to its own radiation, is of the form [2]

$$
\begin{aligned}
N(E)=\frac{1.3 \times 10^{-21}}{d^{2}} Z^{2} & T^{-0.5} \exp (-E / k T)[\bar{g}(E, T)+f(E, T)] \times \\
\times \int N_{e}^{2} \mathrm{~d} V \text { ergs cm } & -2 \mathrm{~s}^{-1} \mathrm{keV}^{-1} \text { at Earth }
\end{aligned}
$$

distance, where $N_{e}$ is the electron density, $E$ the photon energy and $k$ is Boltzmann's constant. $\mathrm{d} V$ is an element of emitting volume and $\int N_{e}^{2} V$ the 'emission integral'. The expression has been simplified by taking a mean value $Z^{2}$, which for a solar abundance is $\sim 1.6$. The two terms in the squared bracket account respectively for the bremsstrahlung radiation, where a free-free Gaunt factor

$$
\vec{g}(E, T)=(E / k T)^{-0.3}
$$


applicable to low $Z$ plasmas is proposed after Rappaport [3], and an empirical function

$$
f(E, T)=\bar{g}(E, T)\left[\left(\frac{88}{E}\right) 0.33 k T-1\right]^{-1}
$$

which approximates the total recombination emission for a gas of the assumed chemical abundance.

Substituting (2) and (3) in Equation (1) gives the complete thermal continuum spectral function [2]

$$
\begin{aligned}
& N(E)=1.4 \times 10^{14} d^{-2} E^{-0.3} T^{-0.2} \exp [-E / k T] \times \\
& \times\left[1-\left(\frac{E}{88}\right) 0.33 k T\right]^{-1} \int N_{e}^{2} \mathrm{~d} V \text { ergs cm }{ }^{-2} \mathrm{~s}^{-1} \mathrm{keV}^{-1} \text { at Earth distance. }
\end{aligned}
$$

It is often convenient and satisfactory to compare available cosmic $\mathrm{x}$-ray spectra with a simpler form of Equation (4). Two commonly used simplifications are

$$
\bar{g}\left(E_{1} T\right)=1.0 \text { and } f(E, T)=0
$$

and

$$
\bar{g}\left(E_{1} T\right)=(k T / E)^{0.3} \text { and } f(E, T)=0 .
$$

From a comparison with observed spectra, a near-exponential spectral shape in the $x$-ray region may be interpreted to yield a value for the plasma temperature while the measured intensity then gives $N_{e}^{2} V / d^{2}$. If the x-ray source has been identified, then data at other wavelengths may allow a complete solution for $N_{e}, V$ and $d$ (see, for example, discussion on Sco X-1 in Section 2).

Emission lines from partly ionised stages of the heavier elements ( $\mathrm{O}$ to $\mathrm{Fe}$ ) will not greatly affect the overall spectral distribution for $k T>1 \mathrm{keV}\left(T>1.2 \times 10^{7} \mathrm{~K}\right)$, although the observation of specific lines are of considerable potential value in confirming the thermal nature of a source and providing direct information on its physical parameters. Again, this may be done by comparing an observed line spectrum with theory. With the assumptions of upper level population principally by electron collision, with a consequent photon emission, a corresponding oscillator strength of $f_{i j}$, the $\bar{g}$ approximation for collisional excitation of an ion, leads [4] to a predicted line flux, from an element of relative abundance $A_{z}$, of

$$
\begin{aligned}
& F_{i j}=1.8 \times 10^{-16} \bar{g} f_{i j} d^{-2} \int G\left(T_{e}\right) N_{e}^{2} \mathrm{~d} V \\
& \operatorname{ergs} \mathrm{cm}^{-2} \mathrm{~s}^{-1} \text { at Earth distance. }
\end{aligned}
$$

This process, again, is mainly dependent on $T$, the temperature dependent parts of (5) being given by an 'emission function' (see Figure 2)

$$
G\left(T_{e}\right)=T_{e}^{-0.5} \frac{N \text { ion }}{N_{z}} 10^{\left(-5040 W_{i j} / T_{e}\right)} .
$$

In Equation (5) $\vec{g} \sim 0.3, f_{i j}$ is the oscillator strength and is reasonably well known for 


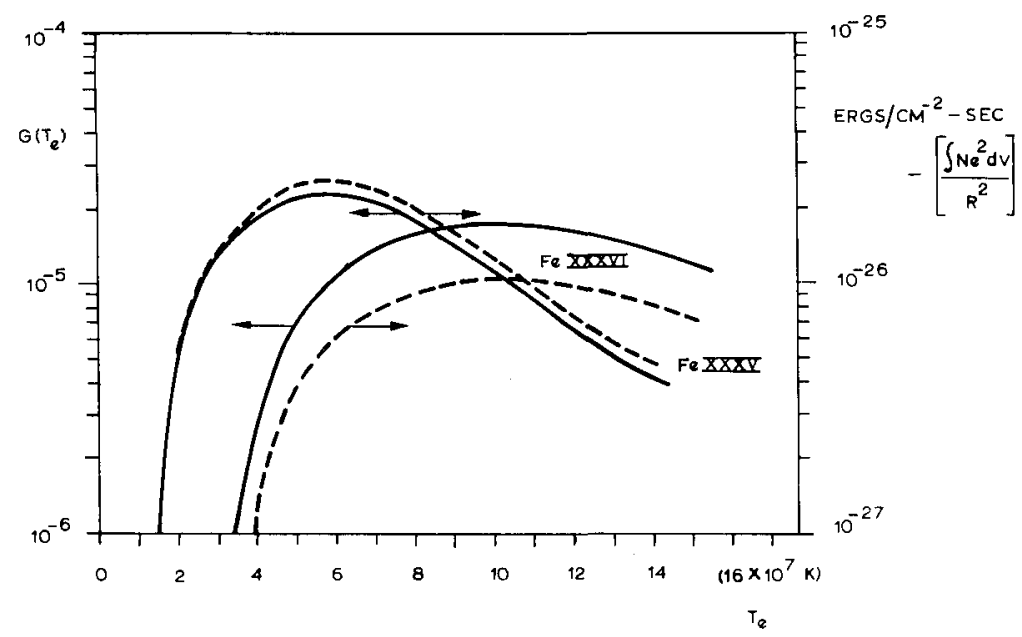

Fig. 2. Computed emission functions (see text) and flux per unit emission measure and steradian for the resonance lines of Fe XXv $\left(1 s^{2}{ }^{1} S-1 s 2 p^{1} P\right)$ and Fe XXvI $(\mathrm{L} \alpha)$. For a thermal source with 'cosmic element abundance' these iron emission lines may be the strongest of all over the wide range of temperatures indicated by presently known cosmic $\mathrm{x}$-ray sources.

the dominant (He- and $\mathrm{H}$-like ion spectra) and $W_{i j}$ is the corresponding excitation potential. Though no emission lines have been clearly established in cosmic $\mathrm{x}$-ray spectra to date, the anticipation of positive fluxes from forthcoming satellite-borne crystal spectrometers (of the Leicester Group on UK-5, Columbia University on OSO-I and the Utrecht-MIT - AS and E collaboration on the Netherlands satellite) offer the exciting prospect of obtaining $T_{e}, N_{e}$ and $V$ from inter-line comparisons, together with relative abundances of the heavier elements. If line profiles can be measured, additional information of $T_{\mathrm{ion}}$, macroscopic mass movement, red-shift and optical depth effects may also become available. For the present review, however, such interpretations remain only enticing possibilities of the future.

\section{B. NON-THERMAL}

A second group of cosmic x-ray spectra appear better fitted with a power-law distribution and are generally, though not definitively, interpreted as evidence for either synchrotron or inverse Compton radiation. In both cases a population of relativistic electrons is required and a typical power law spectrum assumed (or sometimes deduced from the non-thermal radio spectrum of the source, when this is known).

For an electron number spectrum $N(E) \propto E^{-\beta}$ interaction with a magnetic field of $B$ gauss will produce a synchrotron radiation spectrum with power law index $\alpha=$ $=0.5(\beta+1)$, electrons of energy $E$ contributing principally near $6 \times 10^{-9} B\left(E / m c^{2}\right)^{2}$ $\mathrm{eV}$. Thus, for example, electrons of $E \sim 10^{13} \mathrm{eV}$ will generate kilovolt x-rays in a field $B \sim 10^{-3} \mathrm{G}$. A common problem with the extension of the synchrotron process to $\mathrm{x}$-ray energies is that the electron lifetimes are very short. The energy loss rate is $\mathrm{d} E / \mathrm{d} t \sim 10^{-3} B^{2}\left(E / m c^{2}\right)^{2} \mathrm{eV} \mathrm{s}^{-1}$ giving for the above case a lifetime $t_{\mathrm{s}} \sim 60$ days. 
The inverse Compton process, a favourite contender for the isotropic background $\mathrm{x}$-ray production, involves the scattering of low energy photons by relativistic electrons. Energy is transferred in the interaction from the electron to the photon, the energy gain being proportional to $\sim 1.3\left(E / m c^{2}\right)^{2}$. Thus, kilovolt $\mathrm{x}$-rays may be produced in the scattering of microwaves $(3 \mathrm{~K})\left(\varepsilon \sim 10^{-3} \mathrm{eV}\right)$ by $5 \times 10^{8} \mathrm{eV}$ electrons, or of starlight photons $(\varepsilon \sim 2.5 \mathrm{eV})$ by $2 \times 10^{7} \mathrm{eV}$ electrons.

The lifetime of a relativistic electron against inverse Compton losses is $t_{c} \sim 7 \times 10^{11}$ $\left(E / m c^{2}\right) W \mathrm{yr}$, where $W$ is the energy density of the radiation field. For the microwave photons in intergalactic space $W \sim 0.3 \mathrm{eV} \mathrm{cm}^{-3}$ and $t_{c} \sim 2 \times 10^{9} \mathrm{yr}$, short compared to a Hubble time $\left(\sim 10^{10} \mathrm{yr}\right)$. A competing process, such as adiabatic expansion in radio galaxies, would give rise to a break in the electron spectrum, which would then appear as a similar break in the $x$-ray spectrum. Such considerations are relevant to the discussion of the isotropic x-ray background in Section 6.

\section{Scorpius $X-1$}

This first-seen and still brightest of the intense Galactic x-ray sources, identified [5] in 1966 with a 12.5 magnitude star, has received more attention than any other from both $\mathrm{x}$-ray and ground-based astronomers. Figure 3 summarises a selection of spectral data on Sco X-1 from 0.25 to $80 \mathrm{keV}$.

Though variability in both the $x$-ray intensity and spectrum are established [6] the majority of published data between 1 and $20 \mathrm{keV}$ yield a good fit to an exponential spectrum with $k T \sim 4 \mathrm{keV}$. Below $1 \mathrm{keV}$ there is evidence [7] for a variable degree of photoelectric absorption. Assuming Sco X-1 to lie at a distance of 200-500 pc from Earth, a range indicated [8] by most of the optical evidence, a (reasonable) column density of $7 \times 10^{20} \mathrm{H} \mathrm{cm}^{-2}$ would give unit absorption depth at $E=0.8 \mathrm{keV}$. However, if variability in the observed $x$-ray cut-off is confirmed then at least some part of the absorption must be due to relatively cool gas surrounding the source itself.

Comparison of the $\mathrm{x}$-ray spectrum with observations of Sco X-1 in the visible and infra-red have led to important deductions about the physical nature of the $\mathrm{x}$-ray source. Reference to Equation (1) shows that for $k T \sim 4 \mathrm{keV}$ the spectrum of an optically thin source would be exponential at x-ray energies and then nearly flat down to the optical and longer wavelength bands. Examination of Figure 4 shows in fact the optical and infra-red points fall well below the optically thin spectrum. Interstellar reddening is insufficient to account for the lack of low energy photons. Instead, these data appear more consistent with an optically thick source and in the near infra-red the spectrum has the Rayleigh-Jeans $\left(v^{2}\right)$ form. Neugebauer et al. [9] have assumed a spherical plasma cloud of radius $R$ and temperature $T$ at a distance $d$ from Earth. Then, from the black-body emission function applied to their infra-red data, they obtain

$$
\frac{R^{2} T}{d^{2}}=10^{-17} \mathrm{~K} .
$$




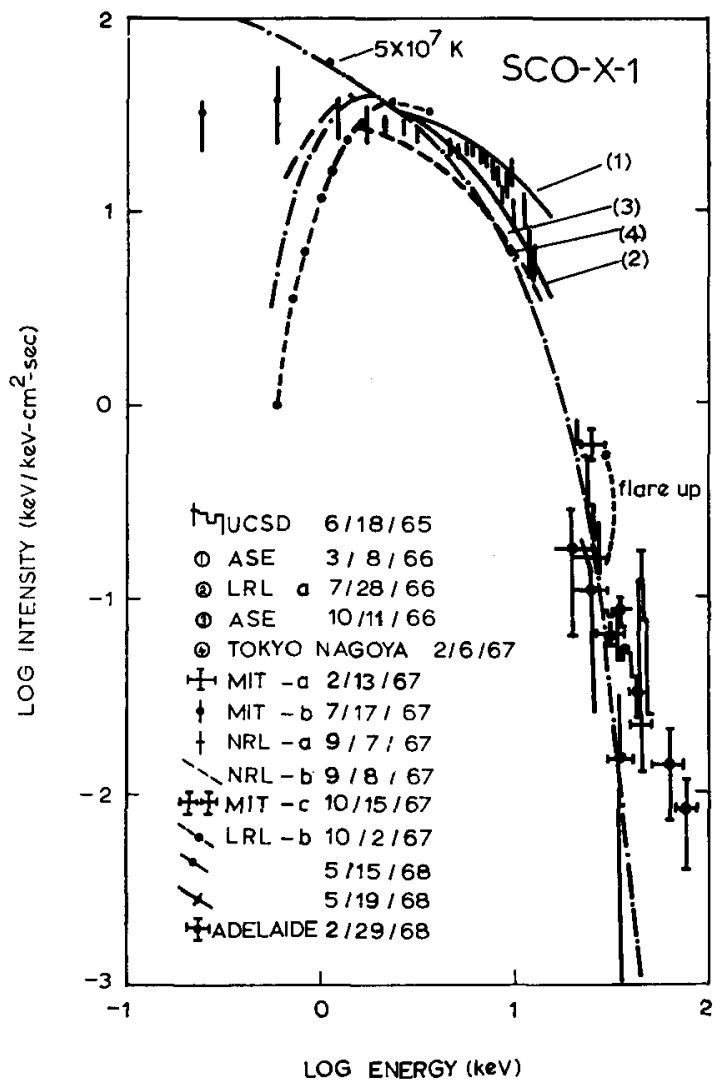

Fig. 3. Summary of most of the published low resolution x-ray spectra of Sco X-1. The dash-dot curve is the exponential spectrum expected for the thermal radiation of an optically thin plasma at a temperature of 50 million K. (reference Equation (4a) in text). Note the typical deviation from a simple exponential spectrum above $20-30 \mathrm{keV}$. The turn-over below $1 \mathrm{keV}$ is generally interpreted as absorption in the source or the interstellar gas.

The $1-20 \mathrm{keV} \mathrm{x}$-ray spectrum typically yields

$$
\frac{N_{e}^{2} R^{3}}{d^{2}}=10^{17} \mathrm{~cm}^{-5}
$$

For $T=5 \times 10^{7} \mathrm{~K}$ and $d=500 \mathrm{pc}$, limiting values of $R \sim 7 \times 10^{8}$ and $N_{e} \sim 3 \times 10^{16} \mathrm{~cm}^{-3}$ are obtained from Equations (2) and (3).

For such a model the long wavelength opacity is mainly due to free-free absorption, given by

$$
\tau_{\mathrm{ff}}=0.02 g_{\mathrm{ff}} \frac{N_{e}^{2} R}{v^{2} T^{3 / 2}} \sim 10^{29} v^{-2}
$$

and the effective optical depth may be approximated by $\tau^{*}=\left(3 \tau_{\mathrm{ff}} \tau_{\mathrm{es}}\right)^{1 / 2}$ where $\tau_{\mathrm{es}}$, the electron scattering thickness, is $6.7 \times 10^{-25} N_{e} R$. For the above Sco X-1 thermal model, $\tau^{*}$ runs from 2-7 over the optical spectrum. 


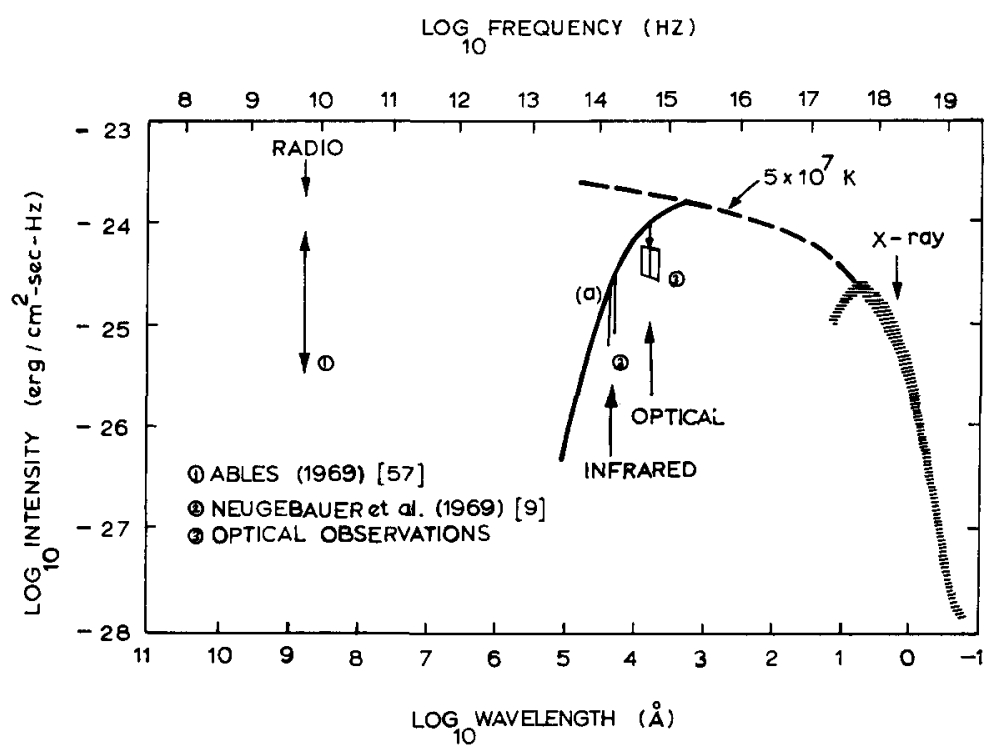

Fig. 4. Composite electromagnetic spectrum of Sco X-1 from $\mathrm{x}$-ray to radio wavelengths. The thermal radiation of an optically thin source at 50 million $\mathrm{K}$ is shown as a dashed line, taking an energy-dependent Gaunt factor (Equation (4b)) into account. The full line ' $a$ ' indicates the improved fit to the optical and infra-red measurements when allowance is made for scattering in a compact source (see [9] of text). The additional effect of interstellar extinction in lowering the observed visible points (3) is shown by the short vertical arrow.

It has often been said in recent years that the definitive test for a thermal model for Sco X-1 would be the search for X-ray line emission. Recently three unsuccessful attempts have been made, using Bragg crystal spectrometers on attitude controlled rocket launches. In March 1970 and again a year later the Leicester Group obtained $(3 \sigma)$ upper limits, for the equivalent width of a narrow resonance line of $\mathrm{Fe} \mathrm{xxV}$ at $6.65 \mathrm{keV}$, of $47 \mathrm{eV}$ and $25 \mathrm{eV}\left(10^{-9} \mathrm{erg} \mathrm{cm}^{2} \mathrm{~s}^{-1}\right)$ respectively [10,11]. A proportional counter detector on the second flight yielded a $3 \sigma$ upper limit near $6.6 \mathrm{keV}$ of $60 \mathrm{eV}$, and since this detector would merge the resonance, intercombination and forbidden lines of Fe XXV and $\mathrm{L} \alpha$ of Fe XXVI and any nearby satellites, this upper limit is slightly more restricting than the high resolution crystal measurement [11]. In comparison, an optically thin plasma of solar iron abundance $\left(4 \times 10^{-5} \mathrm{H}\right)$ at the measured temperature of the second flight (viz. $5.5 \times 10^{7} \mathrm{~K}$ ) would yield a line strength of $400 \mathrm{eV}$. Previous proportional counter spectra had shown marginal evidence [12] (at $3.25 \sigma$ ) of a line of $1-2 \times 10^{-9} \mathrm{erg} \mathrm{cm}^{-2} \mathrm{~s}^{-1}$, an upper limit [13] of $5 \times 10^{-9} \mathrm{erg} \mathrm{cm}^{-2} \mathrm{~s}^{-1}$ and a positive flux [14] at $(6 \sigma)$ of $(7 \pm 4) \times 10^{-9} \mathrm{erg} \mathrm{cm}^{-2} \mathrm{~s}^{-1}$. Only the last mentioned observation, by the Lockheed Group, appears to be in disagreement with the other data. In fact, the compact, high density model derived from the optical and infra-red data [9] appears capable of explaining the non-observation of the Fe xxv lines. Thus a photon originating near the centre of the spherical plasma model would experience $\sim \tau_{\mathrm{es}}^{2}$ Compton scatterings in a 'random walk' passage to the edge of the source. On 
each interaction an energy transfer of $\sim 10 \%$ would occur between the photon and electron. The reduction of at least a factor 15 required by the second Leicester observation implies an electron scattering optical depth $\tau_{\mathrm{es}} \geqslant 3$, when the additional effect of resonance line absorption is taken into account [15].

The remaining attempt to detect line emission was by the Columbia Group, who searched for L $\alpha$ of S XVI at $2.6 \mathrm{keV}$. They obtained a $3 \sigma$ upper limit [16] to a narrow line of $6.7 \mathrm{eV}$, a factor of 3 less than originally expected for an optically thin source at the measured $8 \times 10^{7} \mathrm{~K}$ and a sulphur abundance of $1.6 \times 10^{-5} \mathrm{H}$. However, a reestimation [15] of the expected line, taking into account photoionisation by the strong $\mathrm{x}$-ray continuum of Sco X-1 together with the effects of scattering and line absorption, has reduced the expected S XVI L $\alpha$ flux by a factor $\sim 200$.

In summary, the present upper limits for a narrow Fe xxv line imply $\tau_{\text {es }} \geqslant 3$ while the proportional counter data (excluding that of Lockheed) require the rather larger value of $\tau_{\text {es }} \geqslant 6$. These results are consistent with the infra-red optical data. It is important to note that the continuum shape in the $x$-ray region, and therefore the derived temperature, will also be changed if $\tau_{\mathrm{es}}$ is large. While at small $\tau_{\mathrm{es}}$ the x-ray spectrum can often be characterised by a unique temperature, the exponential shape will show significant deviations at larger $\tau_{\text {es }}$. In effect, photons are transported towards $2.7 k T_{e}$. Loh and Garmire have considered the radiative transfer problem in some detail [17] and examples of their results are shown in Figures 5 and 6 . For

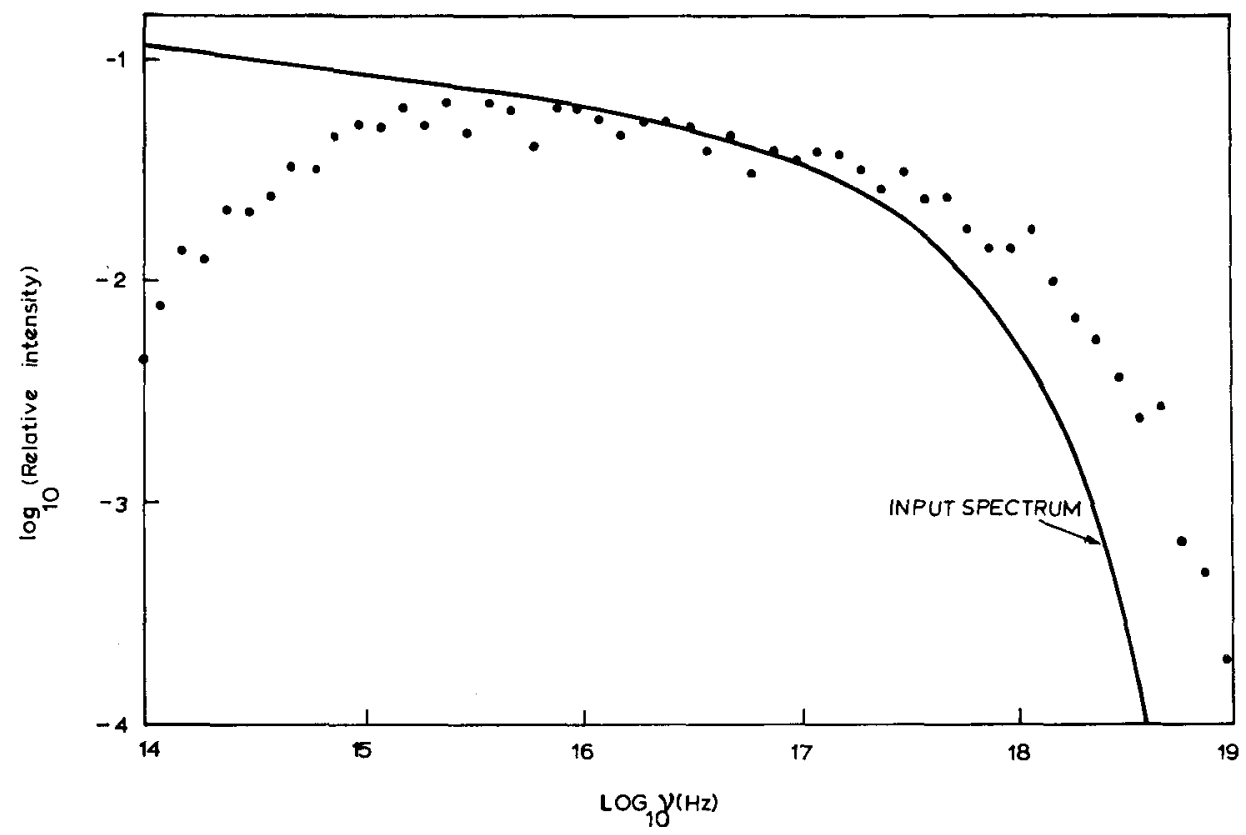

Fig. 5. The effect of opacity in the compact plasma source model of Sco X-1 on the photon continuum. The figure is taken from [17] in the text and depicts the calculated distortion of an input spectrum of 50 million $\mathrm{K}$ in being emitted from a spherical plasma of $R=4.4 \times 10^{8} \mathrm{~cm}$ and $N_{e}=3.4 \times 10^{16} \mathrm{~cm}^{-3}$. 
the case of Sco X-1 the main implication is that temperatures derived from the shape of the continuum have generally been overestimated by up to a factor 2 , though the fact that an exponential fit between $1-20 \mathrm{keV}$ is often a good one indicates the continuum distortion is limited and probably $\tau_{\mathrm{es}}<10$.

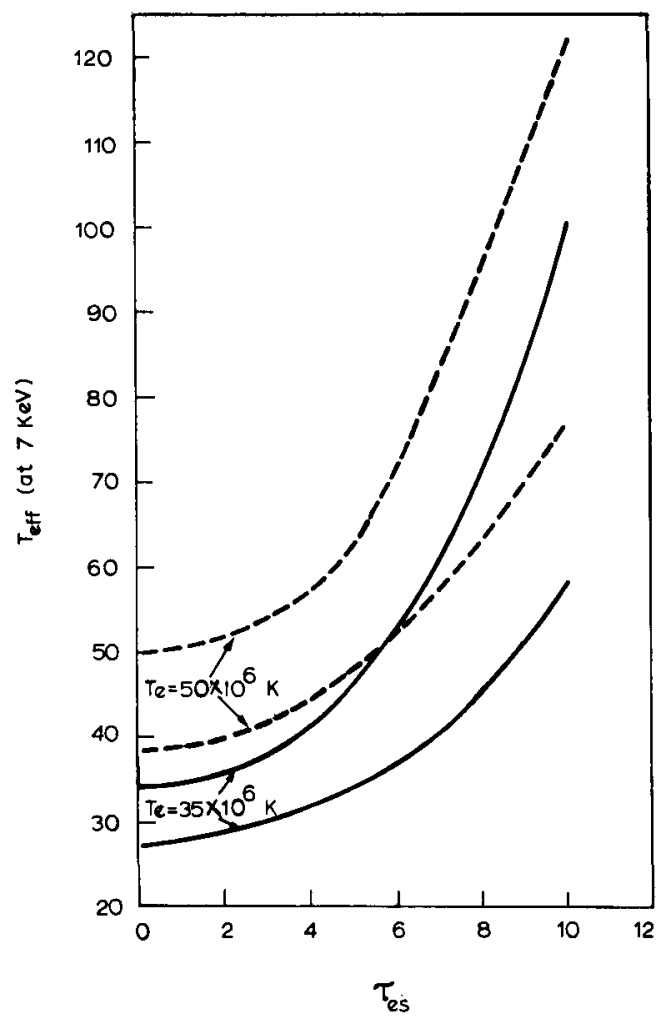

Fig. 6. The increase in effective temperature, measured from the spectral slope at $7 \mathrm{keV}$, as a function of $\tau_{\mathrm{es}}$, for plasmas of kinetic temperature 35 and 50 million $\mathrm{K}$. The upper curve in each case is from a spectral fit including the energy-dependent Gaunt factor and the lower curve from fitting a pure exponential.

A final comment on the X-ray spectrum of Sco X-1 illustrated in Figure 3 is to note the 'harder' or 'hotter' spectrum evident above $30 \mathrm{keV}$. Many of these higher energy measurements [18-20] have been obtained with balloon borne detectors, giving extended observations of a few hours in some cases. These have shown a highly variable spectrum with occasional 'flaring' and characteristic 'temperatures' in excess of $10^{8} \mathrm{~K}$. In fact a power law fit is often better above $20 \mathrm{keV}$ and it has been speculated that the higher energy $\mathrm{x}$-ray spectrum may well be non-thermal in character and perhaps connected with the flaring seen at radio wavelengths. Clarification of this point may come from the extended spectral observations out to $60 \mathrm{keV}$ possible with the MIT counter spectrometer on board the OSO-H satellite to be launched shortly by NASA. 


\section{Other Thermal Sources}

A recent survey of Uhuru spectra showed a number of other Galactic x-ray sources with spectra moderately well fitted by an exponential distribution and apparently falling in two groups, with effective temperatures of 20-70 and >120 million degrees. In general the evidence for a thermal origin is less good in these cases than for Sco X-1 because of the poorer statistics. Even so, the availability of both rocket and balloon data on a few sources have provided a sufficiently large bandwidth to clearly separate power-law and exponential fits and the Nor X-2 spectrum reproduced in Figure 7 is such a case. The increasing evidence for considerable time variability of many Galactic sources casts doubt however on the validity of all such composite spectra, where the different parts have been measured on separate occasions.

A small number of 'soft' $\mathrm{x}$-ray sources visible only below $2-3 \mathrm{keV}$ have been

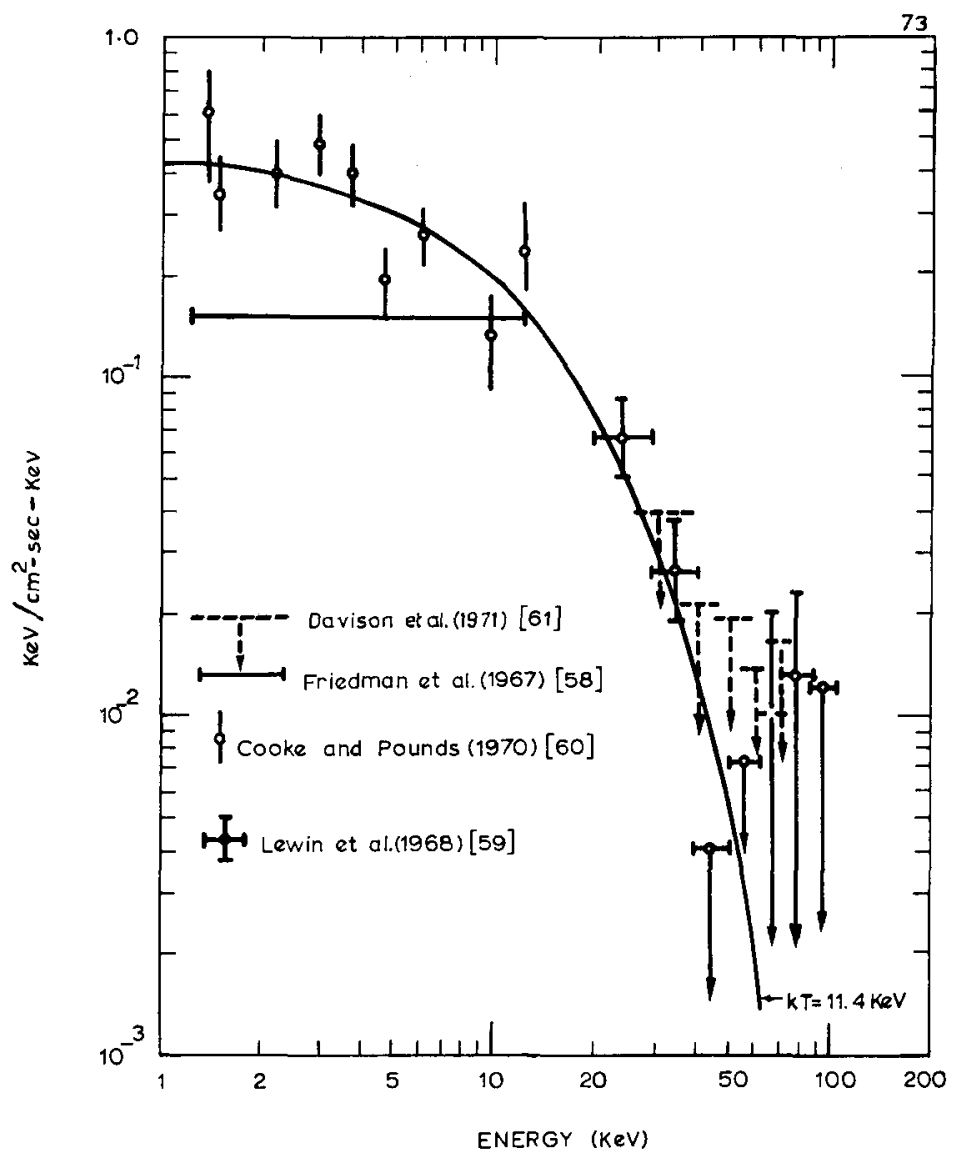

Fig. 7. The X-ray spectrum of Nor X-2. Assuming the source to be not significantly time-variable, the combination of non-simultaneous rocket and balloon data over a wide energy range indicates a thermal spectrum. 
observed in several recent rocket experiments. Most notable are sources apparently associated with the supernova remnants Cygnus Loop, Vela X and Puppis A, each of which is of comparable intensity to Sco X-1 below $1 \mathrm{keV}$. The LRL Group have concluded that their proportional counter spectra on the Vela and Puppis sources are inadequate to indicate a preferred exponential or power law fit [21]. However, for the Cygnus Loop, the AS\&E spectrum (Figure 8) does appear to show a significant excess near $0.65 \mathrm{keV}$ and it has been speculated [22] that this could be due to an unresolved but intense line emission component of $\mathrm{O}$ vII. This would be quite reasonable if the emission from this $50000 \mathrm{yr}$ old supernova remnant was indeed thermal at $T \sim 2$ million degrees and it is interesting to note that the same heliumlike oxygen lines are often the most intense feature in the thermal $x$-ray spectrum of the solar corona. Attempts may be anticipated in the next year or so to observe the x-ray lines from the Cygnus-Loop and clarify this suggestion. In principle such studies will be quite powerful, being capable of yielding values for the temperature, density and chemical composition of the residual supernova hot plasma cloud.

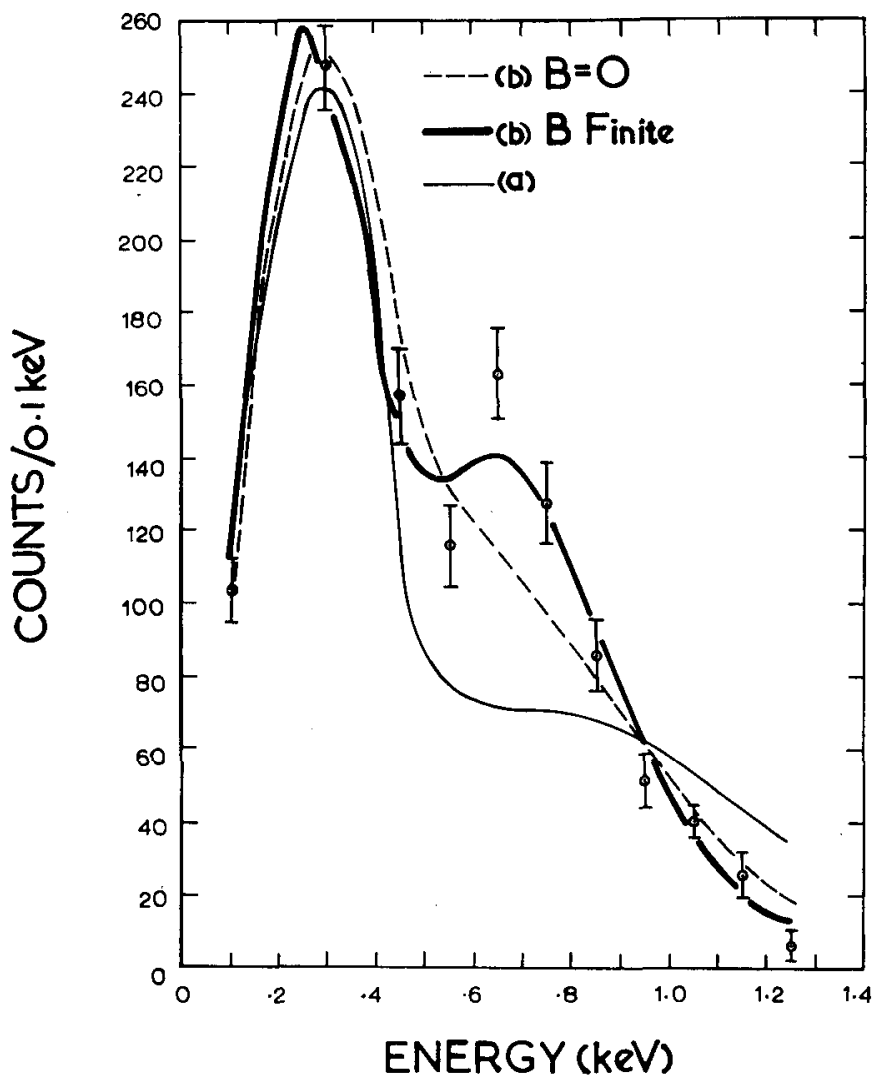

Fig. 8. The count rate spectrum of the Cygnus Loop x-ray source (open circles with $1 \sigma$ error bars) compared with (a) a power law incident photon spectrum and (b) an exponential spectrum with a monoenergetic (line) feature of intensity $B$ at $0.65 \mathrm{keV}$. The best fit to the observed points is obtained with a finite value of $B$ and also some low energy attenuation. 


\section{The Crab Nebula}

The Crab Nebula was the first extra-solar object to be identified [23] with an x-ray source, Tau X-1. The $\mathrm{x}$-radiation is now known to consist of at least two separate components, an extended source $\sim 1.5$ to 2 arc min across and exhibiting a power law spectrum (Figure 9) from below $1 \mathrm{keV}$ to above $500 \mathrm{keV}$, and a somewhat harder power-law spectrum containing up to $20 \%$ of the total x-ray flux from the pulsar

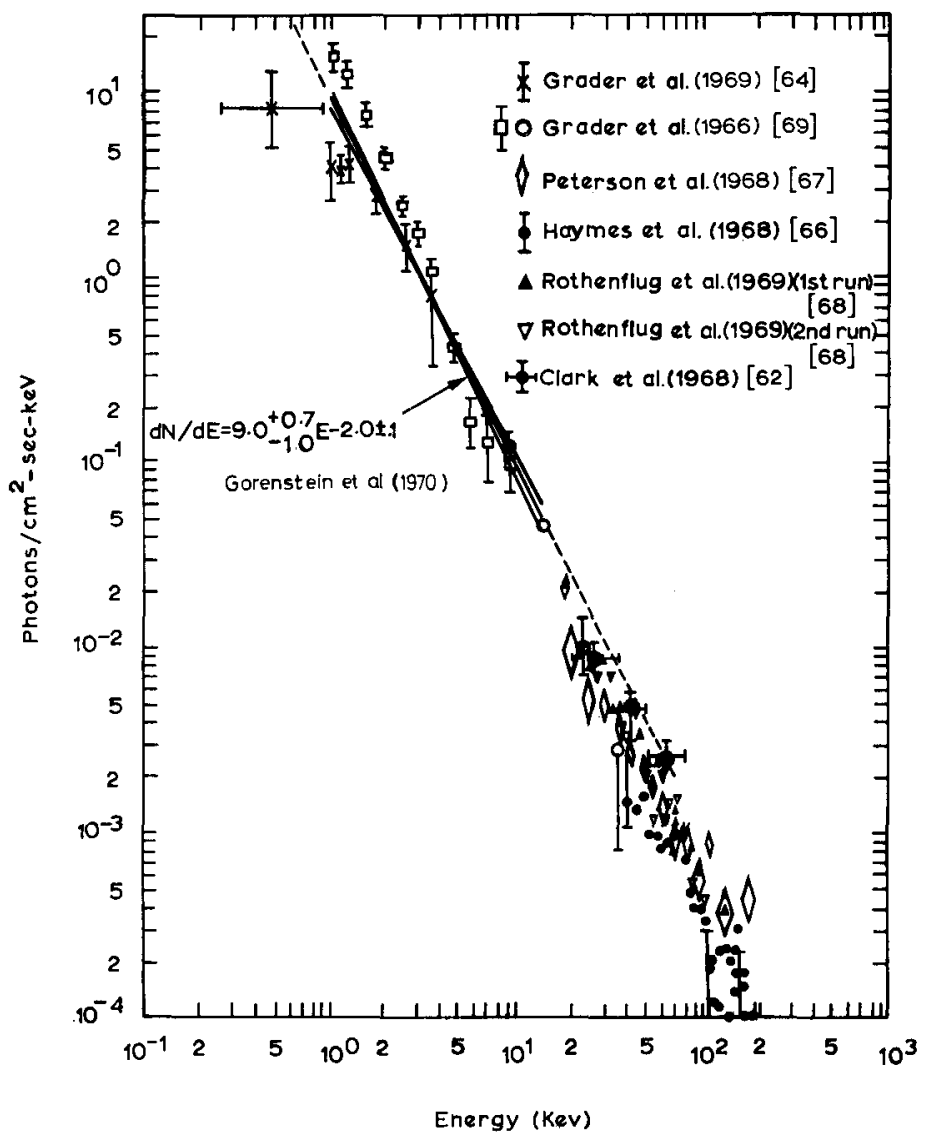

Fig. 9. Summary of spectral data on the total x-radiation of the Crab Nebula. Over a wide energy range a power law spectrum is observed. Recent data referred to in the text showing significant absorption effects below $1 \mathrm{keV}$ are not shown.

NP 0532 (Figure 10). These two are the best available examples of power-law spectra from Cosmic sources. The spectrum of the extended source is well fitted with a power-law index $\alpha$ of 1.1 and this probably connects with the optical continuum and radio spectra, also shown in Figure 10, with a change in the spectral index near the optical region. Support for this view lies in the common interpretation of all three 


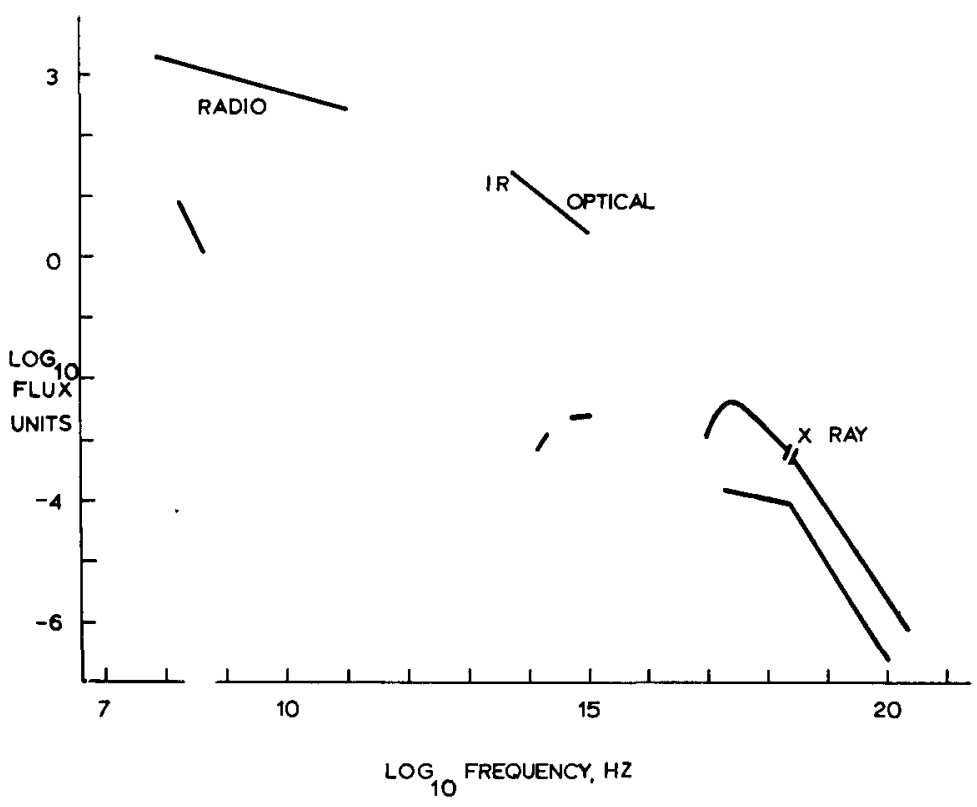

Fig. 10. Composite electromagnetic spectra of the Crab Nebula and the pulsar NPO532 from the $\mathrm{x}$-ray to the radio range. The pulsar data are the lower curves in each spectral band.

spectral bands as electron synchroton radiation and the recent observation that the Crab $\mathrm{x}$-rays are polarised, [24] as are the optical and radio continua, provides some additional confirmation. An interesting consequence of the synchrotron interpretation [25] follows from the application of the formulae in Section 2. Thus, with a magnetic field $B \sim 10^{-3} \mathrm{G}$ (as indicated by optical line splitting and equipartition of energy assumptions) electron energies of $10^{13}-10^{14} \mathrm{eV}$ are required to produce the $\mathrm{x}$-rays and, furthermore, these electrons will have a lifetime only of the order $10^{7} \mathrm{~s}$. This time is, of course, much less than the known (917 yr) age of the Crab Nebula and is also very short compared to the characteristic Fermi acceleration time. A continuous source of new high energy electrons appears to be required and this may well be the central pulsar. As noted earlier the pulsar NP 0532 produces directly a fraction of the Nebula's x-radiation, this component occurring in two sharp pulses which repeat very regularly every $33 \mathrm{~ms}$. With the generally accepted rotating neutron star model for the pulsar, it is not difficult to conceive of ways in which the pulsed $\mathrm{x}$-rays may be produced within or near the 'velocity of light circle' of the neutron star. [26] It is furthermore tempting to suppose that the remainder of the Crab x-radiation is also produced by energetic electrons leaking out into the Nebula. Certainly the extent of the emission region (2-3 light years) is of the right order to be set by the synchrotron radiation lifetime of the relativistic electrons. Also, the similar pulsar and nebula $\mathrm{x}$-ray spectra above $20 \mathrm{keV}$ suggests a strong generic connection. Future observation of the $\mathrm{x}$-ray spectrum as a function of radial distance from the pulsar will test this idea, since this should become steeper (softer) further out. Such spatially resolved 
spectra may also show whether any significant thermal x-ray component is yet evident in this relatively young supernova remnant. Earlier considerations of the whole Crab flux being thermal now seem much less likely after the pulsar discovery and the determination of $\sim 10 \%$ polarisation in the overall x-ray flux. [24]

Observations of the Crab x-ray spectrum below $1 \mathrm{keV}$ have led to some interesting considerations of interstellar absorption. The Crab source is particularly useful in this regard since its extension makes absorption within the source less likely and also its distance is fairly well known $(\sim 2 \mathrm{kpc})$. Published results show some disagreement but indicate the potential of better low energy spectra. Fritz et al. [27] find evidence for a column density of $3.4 \times 10^{21} \mathrm{H} \mathrm{cm}^{-2}$ using the interstellar gas photoelectric cross-section of Brown and Gould [28] and note that this implies twice the gas density indicated by $21 \mathrm{~cm}$ absorption measurements, (with the generally assumed $100 \mathrm{~K}$ spin temperature). They suggest the excess could be due to interstellar grains with a large carbon content. Coleman et al. [29], on the other hand, find a low energy turn-over in the Crab x-ray spectrum which demands significantly less interstellar absorption, but an amount of gas which apparently increases from $7 \times 10^{20} \mathrm{H} \mathrm{cm}^{-2}$ at $1 \mathrm{keV}$ to $16 \times 10^{20} \mathrm{H} \mathrm{cm}^{-2}$ near $0.25 \mathrm{keV}$. If confirmed, this latter result requires either more $\mathrm{He}$, relative to $\mathrm{O}, \mathrm{Ne}$ and $\mathrm{C}$, in the Brown-Gould gas, or the presence of significant molecular hydrogen. At this stage any or all of the above possibilities cannot be ruled out, though the NRL [27] and Wisconsin [29] x-ray results themselves differ by a rather large amount and clearly need confirmation.

\section{Extragalactic Sources}

At the present time some ten extragalactic cosmic $\mathrm{x}$-ray sources have been reported. Four are in the Magellanic Clouds [30] and appear to be of the same type as the most powerful Galactic sources ( $\mathrm{Lx} \sim 10^{38} \mathrm{ergs} \mathrm{s}^{-1}$.) On quite a different luminosity scale are sources apparently associated with the massive elliptical and radio galaxy M87 [31], the Seyfert galaxies NGC 1275 [32,41] and 4151 [32], the brightest visible quasar 3C 273 [33], the powerful radio galaxy Cen-A [33] and the Coma Cluster [34]. Though the intrinsic $\mathrm{X}$-ray powers range from $10^{41} \mathrm{ergs} \mathrm{s}^{-1}$ (NGC 4151) to $10^{46} \mathrm{ergs} \mathrm{s}^{-1}$ (3C 173 , if at $630 \mathrm{mpc}$ ), these objects are so distant that the received $x$-ray signals are very weak, being at most a few percent of that from the Crab Nebula. Spectral detail is thus generally poor or absent.

M87 has been the most widely studied source and an early spectrum of Adams et al. [35] is reproduced in Figure 11. The agreement with the extrapolated radio spectrum from the bright core of the galaxy was interpreted at that time as evidence for common origin of the radio to $\mathrm{x}$-ray spectrum by synchrotron emission from the well known 'optical jet' in M87. More recent observations [36, 37, 38], however, have revealed a less intense and much steeper $\mathrm{x}$-ray spectrum. This variability, if confirmed, appears to require an extremely compact source, a few light-months or less in extent. Just such a source has been reported in the nucleus of the galaxy from long baseline interferometric radio studies [39]. Furthermore, recent evidence for 
variability of the radio flux from this source has been published [40]. From the $\mathrm{x}$-ray spectrum of Figure 12 a clear choice cannot be made between a thermal source $\left(\mathrm{T} \sim 1.2 \times 10^{7} \mathrm{~K}\right)$ and a power law. It has been speculated [37] that the former interpretation could result from $\sim 10^{-2} M_{\odot} \mathrm{yr}^{-1}$ of gas accreting onto a galactic 'black-hole'. Alternatively the x-rays could arise from inverse Compton scattering of low energy photons in the compact radio object by the same electrons as probably produce the radio photons by the synchroton process. As noted above, a better $\mathrm{x}$-ray spectrum will be of great interest.

Crude spectral information is also available for the apparently extended source in the Coma Cluster, though the published Uhuru data [34] is inadequate for a choice of thermal or non-thermal production mechanism. The best-fit exponential indicates a gas temperature of 70 million degrees and, assuming the radiative efficiency of a

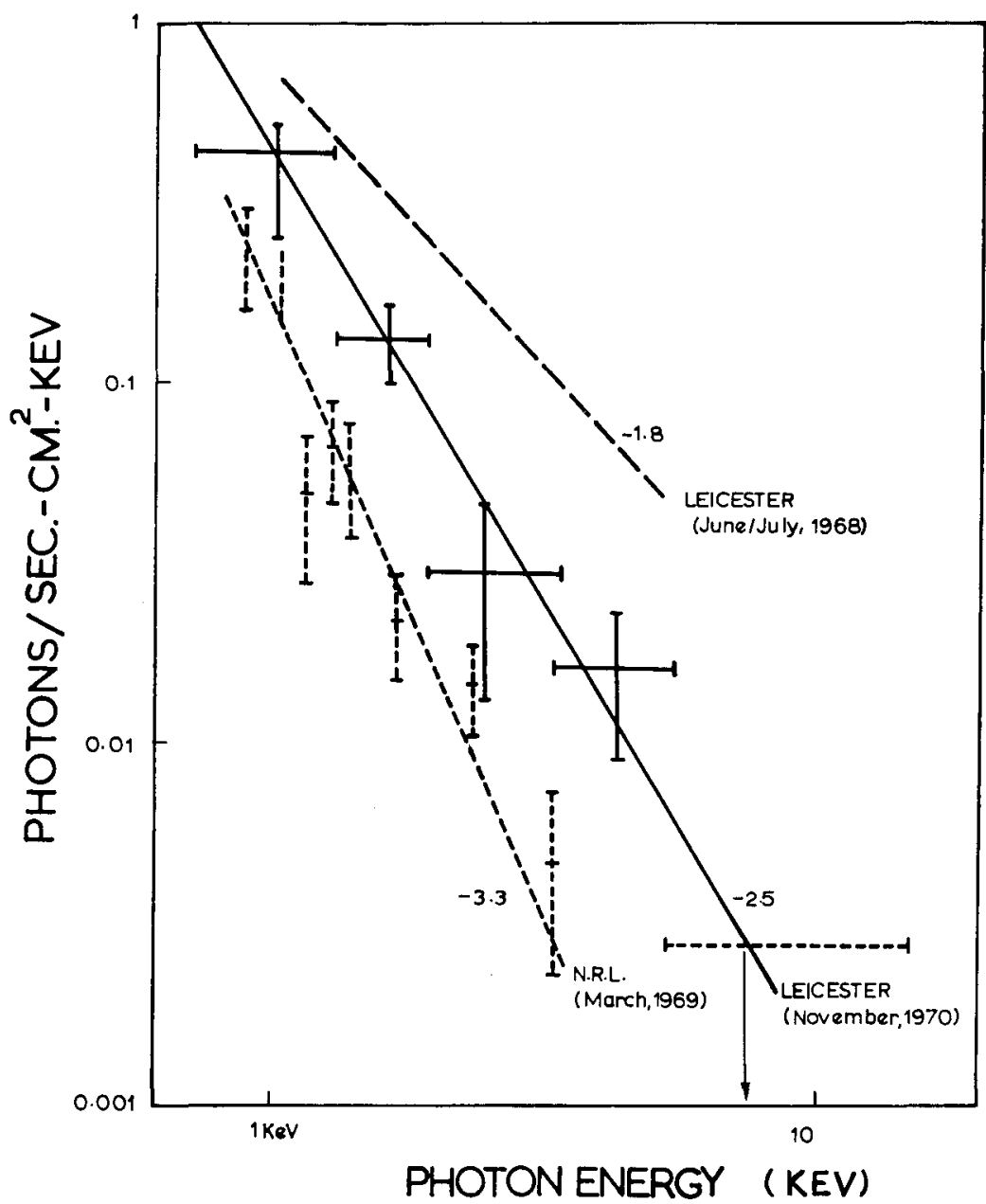

Fig. 11. The x-ray spectrum of M87 measured on rocket flights in 1968, 1969 and 1970. Power law fits are shown in each case. 


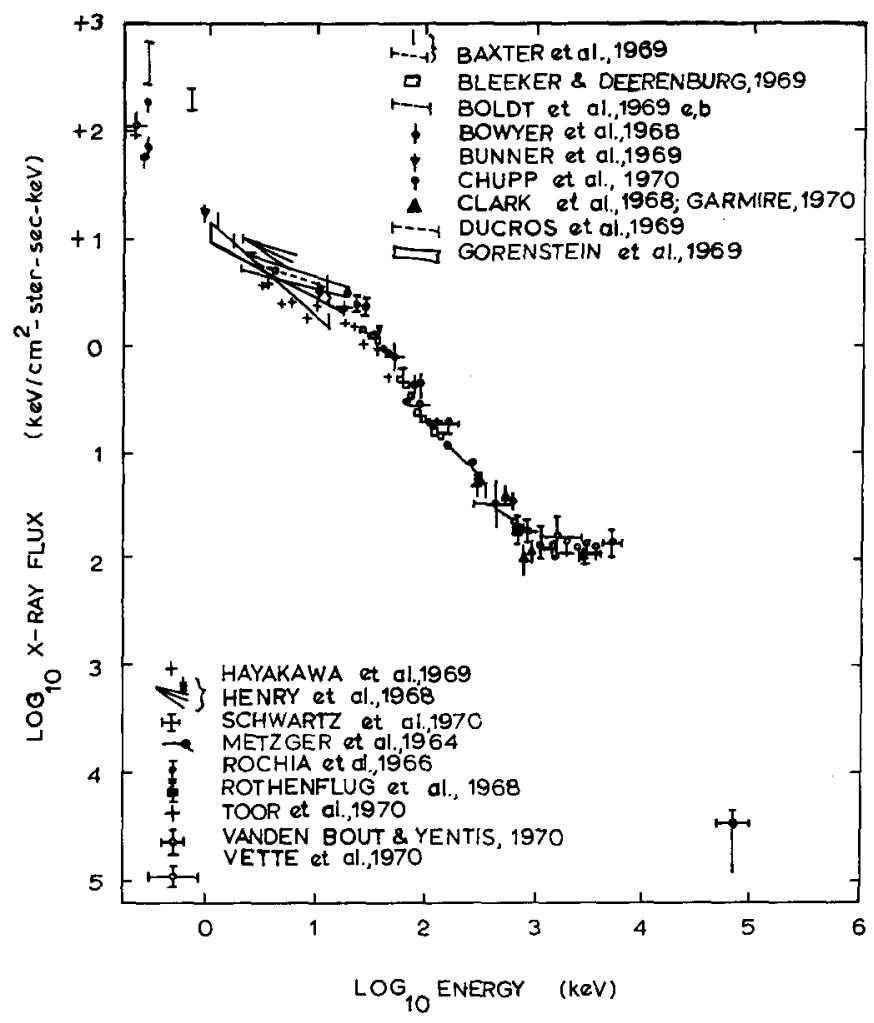

Fig. 12. A summary of available spectral data on the diffuse x-ray background [42].

gas of cosmic abundance, this demands an emitting mass of $3 \times 10^{13} M_{\odot}$. Such a considerable intergalactic gas would be a major component of the Cluster, though nowhere near sufficient for its gravitational containment. However, the $\mathrm{x}$-ray emission detected above $2 \mathrm{keV}$ does not exclude the possibility of a greater amount of cooler gas $\left(T \sim 10^{6} \mathrm{~K}\right)$. Again, a better $\mathrm{x}$-ray spectrum, extended below $1 \mathrm{keV}$, will be crucial. This would serve also to admit or exclude a non-thermal origin for the $\mathrm{x}$-rays. Such a possibility is suggested by the near-coincidence of the $\mathrm{x}$-ray emission region with an extended synchrotron radio source, the $\mathrm{x}$-rays being created by inverse Compton interaction of the radio photons with the relativistic electrons.

\section{Diffuse Background Radiation}

The diffuse and (above $\sim 2 \mathrm{keV}$ ) apparently isotropic x-ray background radiation has been observed over a wide energy range. From $1-1000 \mathrm{keV}$ the spectrum is reasonably well established (Figure 13) and is well fitted with a power law index increasing from 1.7 in the $1-20 \mathrm{keV}$ band to, perhaps, 2.2 at $100-1000 \mathrm{keV}$. The sharpness of this bend or break has been widely discussed recently since it has impor- 
tant implications on extragalactic source models for the diffuse x-rays. [42] Additional observations, all of which need confirmation, are the spectral extension below $1 \mathrm{keV}$, $[43,44]$ with an apparent upturn in shape, an excess flux at 1-5 MeV [45] and a spectral 'bump' near $6.5 \mathrm{keV}$. [46] These spectral features(?) are briefly discussed below.

There seems little doubt that the power-law spectrum from $1 \mathrm{keV}$ to $1 \mathrm{MeV}$ is of extragalactic origin and probably of cosmological importance. The best evidence for this is its isotropy and intensity. Measurements made at high Galactic latitudes, free of known discrete sources, have shown [47] the $2-10 \mathrm{keV}$ intensity to be isotropic to $13 \%$ on a $2^{\circ} \times 8^{\circ}$ scale. If the background is due, as seems likely, to the integrated flux from a large number of distant discrete sources, then this measured isotropy implies a density of at least 12 uniformly bright sources per square degree. The intensity of the background $x$-radiation is remarkable, being equivalent per steradian to about four times the Crab Nebula strength. The intensity and power law spectrum have led to a number of inverse Compton models for the origin of the background radiation. Discrete source models range from the scattering of the intense infra-red fluxes in the nuclei of Seyfert galaxies [48] (and the recent detection of the strong source in NGC 1275 may be the first concrete example), or of radio photons in radio galaxies [49] (such as M87), to the intense radiation of young pulsars occurring in ordinary galaxies. [50] If the NRL observation of a $6.5 \mathrm{keV}$ excess in the background spectrum is confirmed to be of extragalactic origin, then this would be strong evidence for the importance of supernova explosions, since strong fluorescent Fe radiation may be expected to arise. [51] An alternative set of models for the background radiation involve large scale phenomena, such as the inverse Compton scattering of high energy electrons on the universal $(3 \mathrm{~K})$ microwave radiation [52] and thermal bremsstrahlung from a (proposed) dense intergalactic gas. [53]

At present the x-ray spectrum is insufficiently well-determined to assist a choice between these many theories. The present reviewer, at least, knows of no strong evidence for the change in spectral shape between 20 and $200 \mathrm{keV}$ to be a sharp 'break' as sometimes reported in the literature. [54] This is important since, while a break can easily arise in a given discrete source, most probably reflecting a similar feature in the initial electron spectrum, this would be considerably broadened by the red-shift alone if the x-rays are truly cosmological. Thus, inversely, if a sharp break were confirmed the origin of the background would have to be more local. Recent observations [55] have cast serious doubt on the reality of the 1-3 MeV excess, while it seems increasingly clear that the background flux below $1 \mathrm{keV}$ may be of Galactic, Solar System or even (in part) terrestrial origin. [56] Thus it is proposed not to deal with these points in any detail, merely noting the importance of both spectral regions towards obtaining a clearer understanding of the whole x-ray background, when reliable measurements do become available.

\section{References}

[1] Van Speybroeck, L. P.: 1972, this volume, p. 845.

[2] Culhane, J. L. and Acton, L. W.: 1970, Monthly Notices Roy. Astron. Soc. 151, 141. 
[3] Rappaport, S., Bradt, H. V., Naranan, S., and Spada, G.: 1969, Nature 221, 428.

[4] Pottasch, S. R.: 1964, Space Sci. Rev. 3, 816.

[5] Sandage, A. R., Osmer, P., Giacconi, R., Gorenstein, P., Gursky, H., Waters, J. R., Bradt, H., Garmire, G., Sreekantan, B. V., Oda, M., Osawa, K., and Jugaku, J.: 1966, Astrophys. J. 146, 316.

[6] Gorenstein, P., Gursky, H., and Garmire, G.: 1968, Astrophys. J. 153, 885.

[7] Hill, R. W., Grader, R. J., and Seward, F. D.: 1968, Astrophys. J. 154, 655.

[8] Westphal, J. A., Sandage, A., and Kristian, J.: 1968, Astrophys. J. 154, 139.

[9] Neugebauer, G., Oke, J. B., Becklin, E., and Garmire, G.: 1969, Astrophys. J. 155, 1.

[10] Griffiths, R. E., Cooke, B. A., and Pounds, K. A.: 1971, Nature Phys. Sci. 229, 175.

[11] Griffiths, R. E.: 1971, Ph.D. Thesis, Leicester University.

[12] Holt, S. S., Boldt, E. A., and Serlemitsos, P. J.: 1969, Astrophys. J. 158, 155; 1970, in L. Gratton (ed.), 'Non-Solar X- and Gamma Ray Astronomy, IAU Symp. 37, 138.

[13] Fritz, G., Meekins, J. F., Henry, R. C., and Friedman, H.: 1969, Astrophys. J. 156, 33.

[14] Acton, L. W., Catura, R. C., Culhane, J. L., and Fisher, P. C.: 1970, Astrophys. J. 161, 175.

[15] Felten, J. E. and Rees, M. J. (to be published).

[16] Kestenbaum, H., Angel, J. R. P., and Novick, R.: 1971, Astrophys. J. 164, 87.

[17] Loh, E. D. and Garmire, G. P.: 1971, Astrophys. J. 166, 301.

[18] Overbeck, J. W. and Tananbaum, H. D.: 1968, Astrophys. J. 153, 899.

[19] Agrawal, P. C., Biswas, S., Gokhale, G. S., Iyengar, V. S., Kunte, P. K., Manchanda, R. M., and Sreekantan, B. V.: 1971, Astrophys. Space Sci. 10, 500.

[20] Riegler, G. R., Boldt, E., and Serlemitsos, P.: 1970, Nature 226, 1041.

[21] Palmieri, T. M., Burginyon, G., Grader, R. J., Hill, R. W., Seward, F. D., and Stoering, J. P.: 1971, Astrophys. J. 164, 61.

[22] Gorenstein, P., Harris, B., Gursky, H., Giacconi, R., Novick, R., and Van den Bout, P.: 1971, Science 172, 369.

[23] Bowyer, S., Byram, E. T., Chubb, T. A., and Friedman, H.: 1964, Science 146, 912.

[24] Linke, R., Novick, R., and Weisskopf, M. C.: 1971, Bull. Ann. Astron. Soc. 3, 392.

[25] Shklovsky, I. S.: 1966, Sov. Astron. 10, 6.

[26] Shklovsky, I. S.: 1970, Astrophys. J. 159, 77.

[27] Fritz, G., Meekins, J. F., Chubb, T. A., Friedman, H., and Henry, R. C.: 1971a, Astrophys. J. 164,55 .

[28] Brown, R. L. and Gould, R. J.: 1970, Phys. Rev. 1, 2252.

[29] Coleman, P. L., Bunner, A. N., Kraushaar, W. L., and McCammon, D.: 1971, to be published in Astrophys. $J$.

[30] Leong, C., Kellogg, E., Gursky, H., Giacconi, R., Tananbaum, H., and Bishop, J.: 1971, Bull. Am. Astron. Soc. 3, 399.

[31] Bradt, H., Mayer, W., Naranan, S., Rappaport, S., and Spada, G.: 1967, Astrophys. J. 150, 199.

[32] Gursky, H., Kellogg, E. M., Leong, C., Tananbaum, H., and Giacconi, R.: 1971, Astrophys. J. $165,43$.

[33] Kellogg, E., Gursky, H., Leong, C., Schreier, E., Tananbaum, H., and Giacconi, R.: 1971, As" trophys. J. 165, 49.

[34] Gursky, H., Kellogg, E., Murray, S., Leong, C., Tananbaum, H., and Giacconi, R.: 1971, Astrophys. $J .167,81$.

[35] Adams, D. J., Cooke, B. A., Evans, K., and Pounds, K. A.: 1969, Nature 222, 757.

[36] Byram, E. T., Chubb, T. A., and Friedman, H.: 1971, Nature 229, 544.

[37] Janes, A. F., Pounds, K. A., Ricketts, M. J., and Rees, M. J.: 1971, Nature Phys. Sci. 230, 188.

[38] Lampton, M., Bowyer, S., Mack, J. E., and Margon, B.: 1971, Astrophys. J. 168, 1.

[39] Cohen, M. H., Moffet, A. T., Shaffer, D., Clark, B. G., Kellermann, K. I., Jauncey, D. L., and Gulkis, S.: 1961. Astrophys. Letters 2, 141.

[40] Graham, I.: 1971, Nature 231, 253.

[41] Fritz, G., Davidsen, A., Meekins, J. F., and Friedman, H.: 1971, Astrophys. J. 164, 81.

[42] Silk, J.: 1970, Space Sci. Rev. 11, 671.

[43] Bunner, A. N., Coleman, P. C., Kraushaar, W. L., McCammon, D., Palmieri, T. M., Shilepski, A., and Ulmer, T. M.: 1969, Nature 223, 1222.

[44] Henry, R. C., Fritz, G., Meekins, J. F., Chubb, T., and Friedman, H.: 1971, Astrophys. J. 163, 73.

[45] Vette, J., Matteson, J. L., Gruber, D., and Peterson, L. E.: 1970, Astrophys. J. 160, 161. 
[46] Shulman, S., Fritz, G., Meekins, J. F., Chubb, T. A., Friedman, H., and Henry, R. C.: 1971, Astrophys. J. 166, 9.

[47] Shwartz, D. A., Boldt, E. A., Holt, S. S., Serlenitsos, P. J., and Bleach, R. D.: 1971, Nature Phys. Sci. 233, 110.

[48] Longair, M. S. and Sunyaev, R.: 1969, Astrophys. Letters 4, 65.

[49] Felten, J. E. and Rees, M. J.: 1969, Nature 221, 924.

[50] Tucker, W. H.: 1970, Astrophys. J. 161, 1161.

[51] Silk, J.: 1971, to be published in Astrophys. J.

[52] Felten, J. E. and Morrison, P.: 1966, Astrophys. J. 146, 686.

[53] Bergeron, J. E.: 1969, Astron. Astrophys. 3, 42.

[54] Schwartz, D., Hudson, H. S., and Peterson, L. E.: 1970, Astrophys. J. 162, 431.

[55] Golenetsky, S. V., Mazets, E. P., Ilinsky, V. N., Aptekar, R. L., Bredov, M. M., Guryan, Y. A., and Panov, V. N.: 1971, Astrophys. Letters 9, 69.

[56] McCammon, D., Bunner, A. N., Coleman, P. L., and Kraushaar, W. L.: 1971, Astrophys. J. $168,33$.

[57] Ables, J. B.: 1969, Astrophys. J. 155, 27.

[58] Friedman, H., Byram, E. T., and Chubb, T. A.: 1967, Science 156, 374.

[59] Lewin, W. H. G., Clark, G. W., and Smith, W. B.: 1968, Astrophys. J. 152, 49.

[60] Cooke, B. A. and Pounds, K. A.: 1970, Nature 229, 144.

[61] Davison, P. J. N., Buselli, G., Clancy, M. C., and Thomas, R. N.: 1971, Astrophys. J. 167, 479.

[62] Clark, G. W., Lewis, W. H. G., and Smith, W. B.: 1968, Astrophys. J. 151, 21.

[63] Grader, R. J., Hill, R. W., Seward, F. D., and Toor, A.: 1966, Science 152, 1499.

[64] Grader, R. J., Hill, R. W., Seward, F. D., and Hiltner, W. A.: 1969, Astrophys. J. 160, 1193.

[65] Gorenstein, P., Kellogg, E. M., and Gursky, H.: 1970, Astrophys. J. 160, 199.

[66] Haymes, R. C., Ellis, D. V., Fishmen, G. J., Kurfess, J. D., and Tucker, W. H.: 1968, Astrophys. J. 151, 9.

[67] Peterson, L. E., Jacobson, A. S., Pelling, R. M., and Schwartz, D. A.: 1968, Can. J. Phys. 46, 437.

[68] Rothenflug, R., Boclet, D., Claisse, J., Durouchou, P., and Rocchia, R.: 1969, Astron. Astrophys. 1, 48.

\section{DISCUSSION}

B. Woodgate: Could the bump at $\frac{1}{2} \mathrm{keV}$ in the Cygnus loop spectrum be explained by absorption at the oxygen edge, rather than emission in the oxygen line at $18.97 \AA$ ?

$K, A$. Pounds: The observations indicate an excess near $0.65 \mathrm{keV}$ whereas $\mathrm{K}$-shell absorption by relatively cool oxygen would cause a deficiency of observed flux on this high energy side of the oxygen $\mathrm{K}$-edge. Therefore, if the energy calibration was good, as the authors claim, emission is clearly preferable to absorption.

C. de Jager: The curious shape of the spectrum of the background radiation suggest a contribution from spectral lines.

$K$. A. Pounds: I imagine you refer to the upturn in the diffuse background spectrum below $1 \mathrm{keV}$ and again above $1 \mathrm{MeV}$. If verified, these spectral features could be due to strong, unresolved line emission. For example, Sciama has recently suggested $O$ vII line emission could be dominant feature at $0.6 \mathrm{keV}$, being produced in the Galactic Halo by accretion of intergalactic gas. Again, an excess in the 1-4 MeV range clearly suggests nuclear $\gamma$-ray line emission, such as has been estimated by Clayton and others from supernovae. 